\title{
Parâmetros comparativos de indicadores bioquímicos plasmáticos de duas populações de quatis (Nasua nasua - LINNAEUS, 1766) com e sem ação antrópica
}

\author{
[Comparative parameters of plasma biochemical indicators of two populations of Coati \\ (Nasuanasua - LINNAEUS, 1766) with and without human action] \\ L.E. Riekehr Júnior ${ }^{2}$, R. Piau Júnior ${ }^{1 *}$, D.D. Gonçalves ${ }^{1}$, R.O. Kunz ${ }^{2}$, C. Cardeal ${ }^{2}$, \\ J.R. Pachaly ${ }^{1}$, Z. S. Cubas ${ }^{3}$, L.G. E. Valle ${ }^{4}$ \\ ${ }^{1}$ Programa de pós-graduação - Universidade Paranaense - Unipar - Umuarama, PR \\ ${ }^{2}$ Centro Universitário Dinâmica Das Cataratas - Foz do Iguaçu, PR \\ ${ }^{3}$ Itaipu Binacional - Refúgio Biológico Bela Vista - Foz do Iguaçu, PR \\ ${ }^{4}$ Faculdade Anglo-Americano - Foz do Iguaçu, PR
}

\begin{abstract}
RESUMO
Os quatis são mamíferos carnívoros da família Procyonidae, são animais onívoros, encontrados nas Américas do Norte, Central e Sul, sendo amplamente distribuídos no Brasil. O objetivo deste trabalho é analisar as alterações nos parâmetros sanguíneos de duas diferentes populações de quatis (Nasua nasua), da região oeste do Paraná, e avaliar a qualidade de vida e a interferência antrópica nos hábitos alimentares desses animais. A análise sorológica foi realizada em duas diferentes populações de quatis (Nasua nasua) que habitam duas unidades de conservação em regiões distintas no município de Foz do Iguaçu. Em uma das populações, foram visualizados cerca de cinquenta indivíduos, os quais se revelam em exposição direta aos seres humanos, e outra população menor, em torno de trinta indivíduos, os quais não possuem exposição direta aos seres humanos, o que, por sua vez, revelou importantes indicativos da qualidade de vida e de saúde desses animais. Foram realizadas avaliações bioquímicas do soro sanguíneo de 10 quatis adultos; cinco animais do grupo 1, população exposta aos seres humanos, e cinco animais do grupo 2, população com menor exposição. Com os resultados obtidos, foram feitas as comparações dos valores bioquímicos entre os grupos e com os valores de referência pesquisados na literatura. Os exames bioquímicos revelaram alterações significativas nos níveis de glicose e proteínas totais e alterações no nível plasmático de triglicerídeos nos quatis capturados na população em contado direto com seres humanos. Conclui-se que a saúde dos animais que possuem exposição direta com seres humanos está sendo afetada pelo consumo de uma dieta rica em carboidratos e gorduras, oriunda de alimentos industrializados fornecidos pelos turistas.
\end{abstract}

Palavras-chave: análises bioquímicas, animais selvagens, sanidade

\begin{abstract}
The coatis, carnivorous mammals of the Procyonidae family, are omnivorous animals, found in North, Central and South America and are widely distributed in Brazil. The objective of this study was to analyze changes in blood parameters in two different populations of coatis (Nasua nasua), of the western region of Paraná, and to evaluate the quality of life and the human interference in eating habits of these animals. Serologic analysis was performed in two different populations of coatis (Nasua nasua) inhabiting two conservation units in different areas in the city of Foz do Iguaçu. In one population, approximately fifty individuals were visualized, revealing themselves in direct exposure to humans as well as another minor population of about thirty individuals, which have no direct exposure to humans, which revealed important indicatives of the quality of life and health of these animals. Biochemical assessments of ten adults coatis serum were perfomred, five animals in group 1, population exposed to humans and
\end{abstract}

Recebido em 16 de agosto de 2016

Aceito em 10 de outubro de 2016

* Autor para correspondência (corresponding author)

E-mail: piau@unipar.br 
five animals of the group 2, population less exposed. With the obtained results, comparisons were made between the groups' biochemical values and the reference values found in the literature. Biochemical tests revealed significant changes in glucose levels and total protein and changes in plasma levels of triglycerides in coatis captured in the population in direct contact with humans. It follows that the health of animals that have direct exposure to humans is being affected by the consumption of a diet high in carbohydrates and fats, coming from processed foods provided by tourists.

Keywords: biochemical analysis, health, wildlife

\section{INTRODUÇÃO}

Os quatis (Nasua sp.) são mamíferos carnívoros da família Procyonidae, são animais onívoros, encontrados nas Américas do Norte, Central e Sul, amplamente irradiados no Brasil. Relatos de estudos comportamentais afirmam que são animais essencialmente diurnos, reproduzem-se uma vez por ano, vivem com seus filhotes em grupos matriarcais de até 30 indivíduos, e os machos, quando se tornam adultos, vivem isolados, somente se aproximando do grupo na época de acasalamento (Reis et al., 2011). A média de vida na natureza pode chegar de sete a oito anos, mas, em cativeiro, como relatado por Denver (2003) pode alcançar, aproximadamente, 17 anos.

Quanto à morfometria, são carnívoros medianos, com, aproximadamente, 73 a $136 \mathrm{~cm}$ de tamanho, aproximadamente $30,5 \mathrm{~cm}$ de altura, pesando entre 3 e $6 \mathrm{~kg}$, e verifica-se que os machos são maiores que as fêmeas (Braddy, 2003). Possuem uma pelagem espessa e densa, de coloração marrom escurecida a preta; as extremidades do corpo, como patas e focinho, possuem uma coloração mais escura; o ventre é amarelado, puxando para o creme (Labate, 2001). A cabeça é fina e alongada, com o nariz ligeiramente arrebitado, grande e pontudo, extremamente flexível, muito adaptado para o forrageio. Os olhos são quase que totalmente frontais; as orelhas são pequenas e arredondadas, com a parte interna de coloração branca. A cauda possui, em média, seis a sete anéis amarelos, e é muito utilizada para manter o equilíbrio; as patas são plantígradas, com cinco dedos (Braddy, 2003).

Conforme Mendes et al. (2010), em quatis com hábitos naturais, na América do Sul, foram identificados 35 itens alimentares na dieta dessa espécie. Entre os alimentos consumidos, a presa mais comum foram os vertebrados, seguidos de invertebrados e material vegetal. Em $34,2 \%$ das amostras fecais, foram encontradas folhas de gramíneas e, em 5\%, sementes de monocotiledôneas.

Estudos de avaliações sorológicas são de grande relevância, pois indicam as alterações na saúde do animal. Por meio das análises dos parâmetros sanguíneos das duas populações observadas, o estudo se baseou no padrão de normalidade apresentado pela população menor desses animais, localizados numa segunda unidade de conservação, a qual atua como população controle por apresentar suas características naturais, como o forrageio do solo em busca de sementes e frutos, predação de invertebrados e pequenos vertebrados terrestres, e, principalmente, por não apresentar interação com seres humanos. Pesquisas sobre parâmetros sanguíneos em quatis são escassas no Brasil, não havendo trabalhos publicados, e em grande parte do continente americano, haja vista a dificuldade de se encontrarem estudos recentes sobre o referido assunto. Estudos publicados no Peru, por Yupanqui et al. (2008), e no México, por Hernandez et al. (2012) foram utilizados como valores de referência. No entanto, isso faz do presente estudo uma referência para futuras pesquisas envolvendo parâmetros bioquímicos com esses animais, os quais possuem uma relação muito próxima com os seres humanos.

Portanto, este estudo tem como objetivo analisar os parâmetros sanguíneos de duas diferentes populações de quatis (Nasua nasua) da região oeste do Paraná e avaliar a qualidade de vida bem como a interferência antrópica nos hábitos alimentares desses animais.

\section{MATERIAL E MÉTODOS}

Este trabalho foi aprovado pelo Comitê de Ética em Experimentação Animal (Cepeea) da Universidade Paranaense (Unipar), sob protocolo $\mathrm{n}^{\mathrm{o}} 25165 / 2014$. 
O estudo foi realizado na porção sudoeste do terceiro planalto paranaense, incluindo apenas os municípios de Foz do Iguaçu e Santa Terezinha de Itaipu. O planalto de Foz do Iguaçu faz parte da subdivisão do terceiro planalto paranaense, situado no extremo oeste do estado do Paraná $\left(25^{\circ} 53\right.$ até $24^{\circ} 21^{\prime} \mathrm{S}$ e $54^{\circ} 43^{\prime}$ até $\left.54^{\circ} 30^{\prime} \mathrm{W}\right)$, entre as bacias hidrográficas Iguaçu e Paraná, com altitude entre 120 e $540 \mathrm{~m}$, apresentando um clima do tipo subtropical úmido, sem estações definidas. Os principais pontos de coleta foram divididos entre duas unidades de conservação (UC), identificadas neste trabalho como (UC-A), Parque de Proteção Integral, localizada entre as coordenadas geográficas $25^{\circ} 05^{\prime}$ até $25^{\circ} 40^{\prime} \mathrm{S}$ e $54^{\circ} 30^{\prime}$ até $54^{\circ} 40^{\prime} \mathrm{O}$, onde os quatis estão expostos à ação antrópica, e (UC-B), Refúgio da Vida Silvestre, localizada entre as coordenadas geográficas $25^{\circ} 26^{\prime} 57^{\prime \prime} \mathrm{S}$ e $54^{\circ} 33^{\prime} 18^{\prime \prime} \mathrm{O}$, onde os quatis não estão expostos diretamente à ação antrópica (Salamuni et al., 2002).

Todos os procedimentos de captura e de manipulação desses animais foram realizados em campo, por médicos veterinários e biólogos, devidamente treinados, causando o menor estresse aos animais. Os períodos de capturas foram estabelecidos pelas Diretorias das UCs, sendo realizados no início da manhã, em que o fluxo de visitantes era menor. Assim, evitaram-se possíveis sensacionalismos negativos por parte dos visitantes, uma vez que os animais eram submetidos a um pequeno estresse, o que poderia ser interpretado como uma forma de agressão aos quatis. Contudo, essa medida restringiu o número de indivíduos na amostra, porque os quatis realizam forrageamento mais intenso durante o maior pico de visitação. Neste estudo, foram contidos, farmacologicamente, 10 quatis adultos, sendo um macho e quatro fêmeas da população em exposição direta com seres humanos (grupo 1), com peso médio de $5,06 \mathrm{~kg}$, e dois machos e três fêmeas da população mais isolada (grupo 2), com peso médio de $4,32 \mathrm{~kg}$, para coleta de sangue.

Foram realizadas contenções físicas em animais adultos utilizando-se luvas de couro e puçá, evitando, assim, machucar o animal, ou até mesmo causar morte por enforcamento. Os animais da UC-A foram atraídos com frutas para próximo dos pesquisadores e, então, capturados com puçá, pela facilidade de aproximação com esses animais. Na UC-B, foram utilizadas armadilhas do tipo tomahawk, com frutas em seu interior, pois os quatis dessa unidade mostraramse mais ariscos à presença humana. Os pesos foram aferidos por meio de balança tipo gancho (marca SUMAX), antes da indução farmacológica. Os animais foram pesados, sendo suspensos com a balança pela rede do puçá, e, em seguida, foi descontado o peso da rede. As análises biométricas foram feitas com a utilização de paquímetro e fita métrica.

Por meio do protocolo de extrapolação alométrica de Pachaly et al. (2006) e Osilheire et al. (2012), utilizando como base o peso do animal, e da constante energética referente ao seu grupo taxonômico, foram calculadas as doses de drogas a serem administradas ao paciente, mediante injeção. $\mathrm{O}$ coquetel de drogas administrado aos indivíduos consistiu, basicamente, de cloridrato de tiletamina e zolazepam ou cloridrato de cetamina e cloridrato de detomidina. Definidas as doses adequadas, as drogas foram administradas por via intramuscular direta.

Depois de anestesiado, cada animal foi mantido sob criteriosa observação clínica. Cada animal avaliado foi identificado por meio de ficha individual, na qual consta o número de identificação do brinco, o sexo, o peso e a biometria.

Para a coleta sanguínea, foram extraídos $3 \mathrm{~mL}$ de sangue da veia jugular. Após a coleta, as amostras foram armazenadas em tubos de ensaio com gel separador, sendo centrifugadas após uma hora, a partir da coleta, durante seis minutos, na velocidade de 3500rpm. O soro sanguíneo extraído foi acondicionado em microtubos de $1 \mathrm{~mL}$ e congelado posteriormente. Os soros foram encaminhados para o laboratório de análises clínicas da Universidade Paranaense para serem processados para análises dos parâmetros bioquímicos, tais como: glicose, triglicerídeos, colesterol total, ureia, ácido úrico, creatinina, proteínas totais, albumina, globulinas, gamaglutamiltransferase, transaminase glutâmicooxalacética, transaminase glutâmico-pirúvica, cálcio, fósforo e ferro (Brasil, 2005).

Os dados foram analisados no programa Bioestat 5.3, utilizando-se ANOVA para os parâmetros bioquímicos entre os grupos. Foi utilizado nível de significância de 5\% (Ayres et al., 2007). 


\section{RESULTADOS E DISCUSSÃO}

Todas as capturas foram realizadas nas primeiras horas do dia, durante o início do forrageio dos quatis, sempre com a presença de médicos veterinários e biólogos que compunham a equipe de pesquisadores. Os animais foram contidos de forma física e farmacológica, ocasionando o menor estresse possível a eles. Posteriormente, foram realizadas as coletas de sangue.

A confirmação nas alterações bioquímicas de glicose, triglicerídeos e proteínas totais, comparadas ao grupo controle e aos valores de referência, mostrou que os quatis que possuem exposição direta com seres humanos estão ingerindo alimentos industrializados ricos em carboidratos e gorduras. Esse tipo de dieta está prejudicando a saúde e a ecologia desses animais, pois eles estão apresentando obesidade, predisposição a diabetes e problemas cardíacos, bem como mudanças em seu comportamento natural.

O habitat natural dos quatis caracteriza-se desde biomas de mata atlântica, pantanal, cerrados, campos, matas ciliares até bosques e regiões limítrofes a esses espaços. Esses animais possuem ótima adaptação às condições ambientais, mudando sua forma de forrageio em diferentes estratos, sem alterar a estrutura da sociedade. Preferem sempre locais que possuem melhores disponibilidades de alimento (Beisigiel,
2001; Labate, 2001; Nunes, 2001). A perda de habitat, a fragmentação e as ações antrópicas podem levar à redução populacional de algumas espécies, processo conhecido como defaunação da comunidade, com ocorrência de algumas extinções endêmicas, bem como mudanças de hábitos de algumas espécies (Galetti e Dirzo, 2013).

A utilização de valores de referência na consideração de resultados de exames laboratoriais como anormais necessita do conhecimento da faixa de variação dos valores em animais sadios. Essas faixas recebem a denominação de "valores normais" ou "de referência". Diferentes métodos para os valores de referência podem ser estabelecidos, no entanto, todos iniciam com a aquisição de amostras de animais de uma população sadia. $\mathrm{Na}$ grande maioria, os animais sadios apresentam comportamento normal para a espécie em questão e não manifestam nenhuma patogenia (Thrall et al., 2015).

Os resultados das análises sorológicas foram divididos em dois grupos: grupo 1 - população em exposição aos seres humanos; e grupo 2 população mais isolada. Quanto à análise bioquímica da glicose plasmática, o grupo 1 apresentou maiores valores com diferença estatística significativa quando comparado com o grupo 2, conforme descrito na Tab. 1 .

Tabela 1. Média \pm erro-padrão das análises sorológicas de glicose, colesterol e triglicerídeos de duas diferentes populações de quatis (Nasua nasua) da região oeste do Paraná, Brasil

\begin{tabular}{lll}
\hline Variáveis & Grupo 1 & Grupo 2 \\
\hline Glicose $\mathrm{mg} / \mathrm{dL}$ & $106,60 \pm 8,65^{\mathrm{a}}$ & $63,80 \pm 2,40^{\mathrm{b}}$ \\
Triglicerídeos $\mathrm{mg} / \mathrm{dL}$ & $52,40 \pm 16,86$ & $32,00 \pm 3,97$ \\
Colesterol total mg/dL & $121,00 \pm 30,98$ & $144,60 \pm 23,42$ \\
\hline
\end{tabular}

Diferença nas letras significa diferença estatística ANOVA ( $\mathrm{P} \ll 0,05)$ entre os grupos (Ayres et al., 2007).

Quanto aos níveis de glicose no plasma, Hernández et al. (2012) encontraram em quatis machos uma média de $80,09 \mathrm{mg} / \mathrm{DL}$ e, para fêmeas adultas, uma média de $74,36 \mathrm{mg} / \mathrm{dL}$. Os animais do grupo 1 apresentaram uma taxa de glicose bem acima desses valores, enquanto os animais do grupo 2 apresentaram valores próximos aos alcançados pelo presente trabalho. Deve-se levar em consideração que as coletas foram realizadas sempre nas primeiras horas do dia, no início do forrageio das populações, a partir das sete horas da manhã. Conforme descrito por Riccó (2004 - artigo não publicado), falsas hipoglicemias podem ser diagnosticadas, uma vez que a glicólise continua sendo realizada in vitro após a coleta de sangue do animal. No entanto, ocorrem discordâncias entre pesquisadores quanto à real capacidade de a glicemia refletir o status energético. Há uma tendência geral de recomendação da avaliação desse parâmetro no perfil metabólico. Dentre os mecanismos que controlam os níveis de glicose, destacam-se os controles endócrinos pelos hormônios insulina e glucagon sobre $\mathrm{o}$ 
glicogênio e dos glicocorticoides sobre a gliconeogênese. A concentração de glicose pode aumentar no estresse crônico. Os altos valores de glicose sanguínea podem estar relacionados ao consumo de carboidratos presentes em alimentos industrializados e também ao nível de estresse relacionado à exposição desses animais aos seres humanos.

Em relação à análise de outro parâmetro bioquímico, os triglicerídeos, pode-se verificar que, apesar de não terem ocorrido diferenças estatísticas entre os grupos, entre os animais do grupo 1 houve taxas de triglicerídeos mais elevadas, como descrito na Tab. 1. Conforme observado por Hernández et al. (2012), a média dos níveis de normalidade para triglicerídeos é de $28,13 \mathrm{mg} / \mathrm{dL}$ para machos e fêmeas de quatis adultos. Para Isis (1999), os valores de referência para quatis adultos é de $34,51 \mathrm{mg} / \mathrm{dL}$. Os valores do grupo $1(52,4 \mathrm{mg} / \mathrm{dL})$ são superiores aos resultados encontrados por esses autores. Ao se compararem os resultados entre os grupos, observa-se que os animais do grupo 1, que estão em exposição direta aos seres humanos, estão consumindo alimentos com maior teor de carboidratos e gorduras provenientes de alimentos industrializados e os animais do grupo 2 estão como os níveis de triglicerídeos dentro dos parâmetros normais.

Quanto aos níveis de colesterol, não ocorreram diferenças significativas entre os grupos. Esses níveis estão abaixo dos valores de referência, que são de 220,07mg/dL (Isis, 1999; Hernández, 2012).

Para as análises de parâmetros bioquímicos renais presentes na Tab. 2, a ureia no grupo 2 apresentou-se mais elevada quando comparada ao grupo 1 .

Tabela 2. Média \pm erro-padrão das análises sorológicas de ácido úrico, ureia e creatinina de duas diferentes populações de quatis (Nasua nasua) da região oeste do Paraná, Brasil

\begin{tabular}{lll}
\hline Variáveis & Grupo 1 & Grupo 2 \\
\hline Ureia $\mathrm{mg} / \mathrm{dL}$ & $25,50 \pm 5,30^{\mathrm{a}}$ & $44,80 \pm 6,24^{\mathrm{b}}$ \\
Ácido úrico mg/dL & $1,20 \pm 0,20$ & $1,62 \pm 0,13$ \\
Creatinina mg/dL & $1,09 \pm 0,06$ & $0,60 \pm 0,25$ \\
\hline
\end{tabular}

Diferença nas letras significa diferença estatística ANOVA $(\mathrm{P}<0,05)$ entre os grupos (Ayres et al., 2007).

Segundo Hernández et al. (2012), os níveis normais para ureia são de $36,04 \mathrm{mg} / \mathrm{dL}$; para Isis (Clinical..., 1999), de 29,93mg/dL. O grupo 2 apresentou um valor acima da normalidade. Enquanto isso, o grupo 1 mostrou-se dentro dos parâmetros descritos. Em dietas com alto teor proteico, há o aumento da quantidade de aminoácidos absorvidos pelo trato gastrointestinal após o catabolismo de proteínas. Dessa forma, quando a quantidade de aminoácidos excede a necessidade nutricional do animal, o excesso é desaminado no fígado. As estruturas de carbono serão utilizadas para a gliconeogênese ou a lipogênese e as aminas formarão a ureia que será eliminada pelos rins (Thrall, 2015). Provavelmente, os animais do grupo 2 tiveram, durante o período de captura, uma dieta mais rica em proteínas, como artrópodes, moluscos e vertebrados.

Quanto aos níveis de ácido úrico, pode-se constatar que não ocorreu diferença significativa entre os grupos, e esses níveis estão próximos aos valores de referência, que são de $1,2 \mathrm{mg} / \mathrm{dL}$ (Clinical..., 1999; Hernández, 2012).

Os parâmetros normais para a creatinina são de $1,39 \mathrm{mg} / \mathrm{dL}$, descritos por Hernández et al. (2012). O grupo 1, apesar de estar abaixo dos parâmetros normais, revelou maior concentração quando comparado com o grupo 2. Provavelmente ambos os grupos podem estar sofrendo perda de massa muscular, ocasionada por uma dieta deficiente em proteínas. Segundo alguns pesquisadores, a produção de creatinina é proporcional à massa muscular do indivíduo; por outro lado, estudos em humanos mostram que a idade e o sexo influenciam na concentração sérica, e não a massa muscular corporal. A creatinina, depois de formada, é excretada do organismo, por via renal, através da filtragem glomerular. A maioria das dietas pode ocasionar a diminuição do teor sérico de creatinina, porque os nutrientes absorvidos induzem a uma elevação, após a refeição, da taxa de filtragem glomerular (Thrall, 2015). 
Nos resultados obtidos para avaliação hepática, contidos na Tab. 3, verificou-se uma diminuição nas proteínas totais, albuminas e globulinas do grupo 1 comparado com o grupo 2 .

Os valores de referência descritos por Yupanqui et al. (2008) mostram $8,0 \mathrm{mg} / \mathrm{dL}$ para proteínas e $3,9 \mathrm{mg} / \mathrm{dL}$ para albumina. E, segundo Hernández et al. (2012), para globulinas, o valor é de $4,1 \mathrm{mg} / \mathrm{dL}$. Em comparação com os valores de referência, o grupo 1 apresentou as taxas de proteínas totais abaixo do padrão, provavelmente, por esses animais possuírem uma dieta rica em carboidratos e gorduras, sendo pobre em proteínas (Yupanqui et al., 2008). O fígado é o principal órgão responsável pela síntese dessas substâncias, estando a produção diretamente relacionada com o estado nutricional do animal. A diminuição nas taxas de proteínas totais no plasma está relacionada a deficiências hepáticas, transtornos renais e intestinais, hemorragias ou deficiência na nutrição (González e Silva, 2006).

Tabela 3. Média \pm erro-padrão das análises sorológicas de transaminase glutâmico-oxalacética (TGO), transaminase glutâmico-pirúvica (GOT), gamaglutamiltransferase (GGT), fosfatase alcalina, proteínas totais, albumina, globulinas, e creatinofosfoquinase de duas diferentes populações de quatis (Nasua nasua) da região oeste do Paraná, Brasil

\begin{tabular}{lll}
\hline Variáveis & Grupo 1 & Grupo 2 \\
\hline Proteínas totais g/dL & $7,36 \pm 0,11 \mathrm{a}$ & $9,42 \pm 0,34 \mathrm{~b}$ \\
Albumina g/dL & $2,94 \pm 0,32$ & $3,14 \pm 0,17$ \\
Globulinas g/dL & $4,42 \pm 0,27 \mathrm{a}$ & $6,27 \pm 0,17 \mathrm{~b}$ \\
Relação albumina/globulinas & $0,69 \pm 0,12$ & $0,50 \pm 0,02$ \\
TGO UI/L & $200,60 \pm 45,38$ & $210,80 \pm 20,86$ \\
TGP IU/L & $87,80 \pm 20,14$ & $98,60 \pm 9,61$ \\
Gama GT U/L & $11,00 \pm 2,88$ & $16,60 \pm 4,83$ \\
Fosfatase alcalina U/L & $29,40 \pm 3,93$ & $33,40 \pm 3,59$ \\
\hline
\end{tabular}

Diferença nas letras significa diferença estatística ANOVA $(\mathrm{P}<0,05)$ entre os grupos (Ayres et al., 2007).

Yupanqui et al. (2008) mostram que os valores de referência para globulinas são de $3,9 \mathrm{mg} / \mathrm{dL}$. Alterações nesses parâmetros foram percebidas para os dois grupos, pois estes apresentaram taxas elevadas de $4,42 \mathrm{mg} / \mathrm{dL}$ no grupo 1 e de $6,27 \mathrm{mg} / \mathrm{dL}$ no grupo 2 , conforme descrito na Tab. 3. Animais de ambos os grupos apresentavam sintomas de uma possível parasitose. As globulinas têm como função o transporte de metais, lipídios e bilirrubina, além do papel na imunidade. São indicadores limitados do metabolismo proteico, com grande importância como indicadores de processos inflamatórios. Dessa forma, quando se depara com altas taxas de globulinas, associam-se os valores às doenças infecciosas ou a vacinações recentes. Por outro lado, alterações nas taxas de globulinas podem ser utilizadas para se analisar a adaptação ao estresse: animais adaptados possuem níveis normais, ao contrário daqueles não adaptados (González e Silva. 2006).
Não se observaram diferenças significativas nos níveis plasmáticos de gamaglutamiltransferase, valor de referência $25 \mathrm{mg} / \mathrm{dL}$; transaminase glutâmico-oxalacética, valor de referência 218 $\mathrm{mg} / \mathrm{dL}$; transaminase glutâmico-pirúvica, valor de referência $221 \mathrm{mg} / \mathrm{dL}$. Quando se compararam os grupos, estes estavam abaixo dos valores considerados de referência para quatis adultos (Clinical..., 1999). Os valores de fosfatase alcalina, que são de $25 \mathrm{mg} / \mathrm{dL}$, estão de acordo com os resultados encontrados por Yupanqui et al. (2008) e Hernandez et al. (2012).

Nas análises sorológicas de cálcio, fósforo e ferro presentes na Tab. 4, não se verificaram alterações significativas entre os grupos. Os valores observados estão dentro dos índices de normalidade descritos na literatura: para cálcio, $8,8 \mathrm{mg} / \mathrm{dL}$; fósforo, $3,9 \mathrm{mg} / \mathrm{dL}$ e ferro, 128,8mg/dL (Hernández et al., 2012). 
Tabela 4. Média \pm erro-padrão das análises sorológicas de cálcio, fósforo e ferro de duas diferentes populações de quatis (Nasua nasua) da região oeste do Paraná, Brasil

\begin{tabular}{lll} 
Variáveis & Grupo 1 & Grupo 2 \\
\hline Cálcio mg/DL & $9,52 \pm 0,59$ & $8,83 \pm 0,39$ \\
Fosforo mg/DL & $6,30 \pm 0,98$ & $5,44 \pm 0,99$ \\
Ferro sérico $\mu \mathrm{g} / \mathrm{DL}$ & $122,76 \pm 34,91$ & $135,82 \pm 15,24$ \\
\hline
\end{tabular}

Diferença nas letras significa diferença estatística ANOVA (p $\ll 0,05)$ entre os grupos (Ayres et al., 2007).

O cálcio é uma das substâncias inorgânicas que possui grande relação com o metabolismo dos animais, estando presente na estrutura óssea, regulação metabólica, coagulação sanguínea, contração muscular, além de atuar na transmissão de impulsos nervosos. O fósforo é um mineral indispensável no processo de mineralização óssea e está presente nos nucleotídeos estruturais de ácidos nucleicos e energéticos, como o ATP. Atua na regulação de enzimas alostéricas e na composição dos fosfolipídios (González, 2000 apud Bockor, 2010 - artigo não publicado). No transporte e na reserva de oxigênio, o ferro é um componente indispensável, agindo no transporte de elétrons, além de estar ligado a diversas enzimas, como a catalase e a aconitase. Trata-se de um íon importantíssimo na formação do grupo heme da hemoglobina, de forma que, quando esse cátion é liberado da hemoglobina, pode ser armazenado no sistema retículo endotelial sob a forma de ferritina e hemossiderina (González e Silva, 2006).

\section{CONCLUSÃO}

A escolha de duas populações diferentes de quatis (Nasua nasua) para realização de avaliações sorológicas laboratoriais mostrou-se eficiente e prática para a comprovação de alterações na qualidade de vida de animais que possuem contato direto com seres humanos, proporcionando-lhes efeitos colaterais gerados por uma dieta irregular. Os animais do grupo 1 apresentaram alterações plasmáticas significativas de glicose e proteínas totais quando comparados ao grupo 2. Com os resultados deste trabalho, pode-se buscar uma reavaliação das estratégias utilizadas para evitar a introdução de alimentos industrializados na dieta cotidiana desses animais, demonstrando a necessidade de uma educação ambiental mais efetiva.

Arq. Bras. Med. Vet. Zootec., v.69, n.3, p.659-666, 2017

\section{AGRADECIMENTOS}

Ao Instituto de Pesquisa e Ambiência Científica (Ipeac) - Unipar, pelo apoio financeiro.

\section{REFERÊNCIAS}

AYRES, M.; AYRES, J.R.M.; AYRES, D.L. et al. BioEstat 5.3: aplicações estatísticas nas áreas das ciências bio-médicas. Belém: [s.n.], 2007. 364p.

BEISIEGEL, B.M. Notes on the coati, Nasua nasua (Carnivora: Procyonidae) in an Atlantic forest área. Rev. Bras. Biol., v.61, p.689-692, 2001.

BOCKOR, L. Indicadores bioquímicos do status nutricional. 2010. 23p. Seminário (Pósgraduação em Ciências Veterinárias) Universidade Federal do Rio Grande do Sul, Porto Alegre.

BRADDY, S. "Nasua nasua" (On-line), Animal Diversity Web. 2003. Disponível em: $<$ http://animaldiversity.ummz.umich.edu/account s/Nasua_nasua.html $>$. Acessado em: 31 out. 2015.

BRASIL. Ministério da Saúde. Agência Nacional de Vigilância Sanitária. Resolução n.302. de 13 de out. 2005. Dispõe sobre regulamento Técnico para Funcionamento de Laboratórios Clínicos. Diário Oficial da União, Brasília, 14 de Outubro de 2005.

CLINICAL pathology records report-ISIS/In house reference values mammals. 1999. Available in: <http://www.worldzoo.org $>$ Accessed in: 10 agost. 2015.

DENVER, M.; FOWLER, M.E.; MILLER, R.E. Procyonidae and Viverridae. Zoo and Wild Animal Medicine, v. 50, p. 515-523, 2003.

GALETTI, M.; DIRZO, R. Ecological and evolutionary consequences of living in a defaunated world. Biol. Cons., v.163, p.1-6, 2013. 
GONZÁLEZ, F.H.D.; SILVA, S.C. Introdução à bioquímica clínica veterinária. Porto Alegre: Universidade Federal do Rio Grande do Sul, 2006.37 p.

HERNANDEZ, M.J.R.; GARCÍA, F.; OMORALES, J.E.M. et al. Hematological and blood chemistry values in a semi-free population of white-nosed Coatis (Nasua narica) in $\mathrm{La}$ Venta Tabasco, Mexico. Act. Zool. Mex., v.28, p.391-400, 2012.

LABATE, A.S. Biology en order Carnivora, family Procyonidae (raccoons, kinkajous). In: FOWLER, M.E.; ZALMIR, C. (Eds.). Biology, medicine and surgery of south American wild animals. Iowa: [Willey] 2001. cap.28.

MENDES, F.R.; MIKICH, S.B.; QUADROS, J. et al. Ecologia alimentar de carnívoros (Mammalia, Carnivora) em remanescentes de Mata Atlântica, sul do Brasil. Biota Neotrop., v.10, p.1-10, 2010.

NUNES, A.L.V. Captive management and restraint in Order Carnivora, family Procyonidae (raccoons, kinkajous). In: FOWLER, M.E.; CUBAS Z.S. Biology, medicine and surgery of south American wild animals (Eds.). Iowa: [University Press], 2001. p.317-322.

OSILHEIRE Jr., J.A.; PEREIRA, R.L.; HERRERA, D. et al. Novo método para contenção farmacológica e anestesia de campo em onças-pintadas (Pantheraonca), empregando a fórmula ZAD (Zoletil/100 + Atropina + Dormiun-V) Relato preliminar. Hora Vet., v.32, p.54-59, 2012.
PACHALY, J.R. Terapêutica por extrapolação alométrica. In: CUBAS, Z.S.; SILVA, J.C.R.; CATÃO-DIAS, J.L. Tratado de animais selvagens: medicina veterinária. São Paulo: Roca, 2006, p.1215-1223.

REIS, N.R.; PERACCHI, A.L.; PEDRO, W.A. et al. Mamíferos do Brasil. Londrina: UEL, 2011. 439p.

RICCÓ, D. Indicadores sanguíneos e corporais de avaliação metabólico-nutricional em ruminantes. 2004. 13f. Seminário (Pósgraduação em Ciências Veterinárias) Universidade Federal do Rio Grande do Sul, Porto Alegre.

SALAMUNI, R.; SALAMUNI, E.; ROCHA, L.A.; ROCHA, A.L. Parque Nacional do Iguaçu, PR: cataratas de fama mundial. In: SCHOBBENHAUS, C.; CAMPOS, D.A.; QUEIROZ, E.T. et al. (Eds.). Sítios geológicos e paleontológicos do Brasil. Brasília: DNPM/CPRM (SIGEP), 2002. p.313-321.

THRALL, M.A. Hematologia e bioquímica clínica veterinária. 2.ed. São Paulo: Roca, 2015. $688 \mathrm{p}$.

YUPANQUI C.C.; LI, O.E.; SILVA, W.S. et al. Perfil bioquímico sanguíneo hepático en coatíes (Nasua nasua) criados en cautiverio. Rev. Invest. Vet. Peru, v.19, p75-78, 2008. 